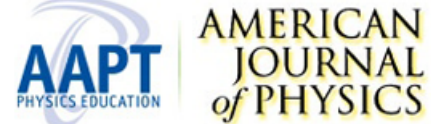

\section{Simple derivation of general Fierz-type identities}

C. C. Nishi

Citation: American Journal of Physics 73, 1160 (2005); doi: 10.1119/1.2074087

View online: http://dx.doi.org/10.1119/1.2074087

View Table of Contents: http://scitation.aip.org/content/aapt/journal/ajp/73/12?ver=pdfcov

Published by the American Association of Physics Teachers

\section{AAPT Re-register for Table of Content Alerts}

\section{Create a profile.}




\title{
Simple derivation of general Fierz-type identities
}

\author{
C. C. Nishi ${ }^{\mathrm{a})}$ \\ Instituto de Física Teórica, Universidade Estadual Paulista, Rua Pamplona 145, \\ 01405-900, São Paulo, SP, Brazil
}

(Received 17 February 2005; accepted 26 August 2005)

\begin{abstract}
General Fierz-type identities are examined and their well-known connection with completeness relations in matrix vector spaces is shown. In particular, I derive the chiral Fierz identities in a simple and systematic way by using a chiral basis for the complex $4 \times 4$ matrices. Other completeness relations for the fundamental representations of $\mathrm{SU}(N)$ algebras can be extracted using the same reasoning. (C) 2005 American Association of Physics Teachers.
\end{abstract}

[DOI: $10.1119 / 1.2074087]$

\section{INTRODUCTION}

The Fierz identities ${ }^{1}$ are frequently used in particle physics to analyze four-fermion operators ${ }^{2}$ such as current-current operators. These reordering identities are used to write a product of two Dirac bilinears ${ }^{3}$ as a linear combination of other products of bilinears with the four constituent Dirac spinors in a different order. Because Fierz identities relate Dirac bilinears, which are objects with well defined transformation properties under the Lorentz group, and Dirac gamma matrices form a representation of the Lorentz group generators, it is not surprising that Fierz identities imply the existence of a basis of four-vectors formed by Dirac bilinears and guarantee its orthogonality and completeness properties. The converse is also possible, that is, recovering the spinor from that basis, which reveals the equivalence between spinor and tensorial representation of various quantities.

Fierz identities are only a particular set of matrix identities, valid for the Dirac bilinears which span the space of $4 \times 4$ complex matrices. General Fierz-type identities can be found for any $N \times N$ real or complex square matrices. The primary aim of this article is to show that any Fierz-type relation can be deduced using just a few ingredients such as the notion of a basis in a vector space and its properties of orthogonality and completeness. The relevant vector space in this case is the vector space of square matrices. For such a vector space, a basis and the orthogonality property between its elements can be defined through an inner product. These ideas are explained in detail in Sec. II for general square matrix vector spaces. Some particularly useful examples involving Pauli matrices [SU(2) algebra], Gell-Mann matrices [SU(3) algebra], and fundamental representations of general $\mathrm{SU}(\mathrm{N})$ algebras are given.

As a particular nontrivial application of these ideas, we will find a straightforward way to deduce the chiral Fierz identities, that is, Fierz identities involving bilinears containing left/right chiral projectors. One way to obtain the chiral Fierz identities is to use the original Fierz identities; one can find very general Fierz identities for usual bilinear in Ref. 4 . However, this procedure may become quite lengthy because it requires expanding the left/right projected bilinears in terms of the usual bilinears, performing Fierz reorderings, and then rewriting them as projected bilinears. Such an approach was adopted in Ref. 5, but will not be pursued here. Instead, the chiral Fierz identities will be deduced by rederiving the Fierz identities using appropriate left/right projected bilinears as a basis (Sec. IV). The simple form of certain chiral Fierz identities is already an indication that a much simpler procedure should exist to derive them.
To exemplify the practical importance of chiral Fierz identities, I will show a situation where the reordering permitted by chiral Fierz identities can help us to better understand and more simply describe a physical system. The obvious area where the chiral Fierz identities can be important is the physics involving the weak interaction, which is a mainly lefthanded interaction. The particular physical system concerns the neutrinos propagating through ordinary matter. Because ordinary matter contains electrons but is absent of muons and tauons, only the electron neutrinos interact with the electrons in matter through the charged-current effective interaction Lagrangian $^{6}$

$$
\frac{4 G_{F}}{\sqrt{2}} \bar{\psi}_{e}(x) \gamma^{\alpha} L \psi_{\nu_{e}}(x) \bar{\psi}_{\nu_{e}}(x) \gamma_{\alpha} L \psi_{e}(x),
$$

where $\psi_{e}, \psi_{\nu_{e}}$ are the fields for the electron and the electron neutrino, respectively, and $L=\left(\mathbf{1}-\gamma_{5}\right) / 2$ is the projector for left chirality. Using a chiral Fierz identity we can rewrite Eq. (1) in the form

$$
\frac{4 G_{F}}{\sqrt{2}} \bar{\psi}_{\nu_{e}}(x) \gamma^{\alpha} L \psi_{v_{e}}(x) \bar{\psi}_{e}(x) \gamma_{\alpha} L \psi_{e}(x) .
$$

This reordered form enables us to describe the influence of nonrelativistic electrons in matter by their average density $2\left\langle\bar{\psi}_{e}(x) \gamma_{\alpha} L \psi_{e}(x)\right\rangle \sim \delta_{\alpha 0} n_{e}$. Such a term leads to an effective interaction acting on electron neutrinos that is different from the interactions acting on other types of neutrinos. Ultimately, it leads to a significant modification of the description of neutrino oscillations, ${ }^{6}$ the phenomenon responsible for the missing solar neutrino problem.

Before we get into the details, let us consider the difference between the expressions in Eqs. (1) and (2) to understand better the nonintuitive nature of the Fierz identity. The spinor fields $\psi_{e}$ and $\psi_{\nu_{e}}$ can be written as complex $4 \times 1$ matrices, while $\bar{\psi}_{e}$ and $\bar{\psi}_{\nu_{e}}$ are complex $1 \times 4$ matrices. The factors between them are $4 \times 4$ matrices and the result is a scalar. In Eq. (1), there is a $4 \times 4$ matrix between $\bar{\psi}_{e}$ and $\psi_{\nu_{e}}$ and another between $\bar{\psi}_{\nu_{e}}$ and $\psi_{e}$ for each $\alpha=0,1,2,3$. What the Fierz identities assure you is that any expression of the form $\bar{\psi}_{1} A \psi_{2} \bar{\psi}_{3} B \psi_{4}$ is equal to $\bar{\psi}_{1} C \psi_{4} \bar{\psi}_{3} D \psi_{2}$ or a sum of similar terms, with $A, B \neq C, D$ in general. Amazingly, the same combination of matrices that enters into Eq. (1) also enters into Eq. (2) as a consequence of a Fierz identity. 


\section{MATRIX VECTOR SPACES AND COMPLETENESS RELATIONS}

A vector space $V$ is a set of elements endowed with two operations: ${ }^{8}$ A sum between elements and a multiplication by numbers (elements of a ring, in mathematical language) such as the real and complex numbers. This vector space is required to be closed under such operations. ${ }^{9}$ The usual $N$-dimensional real space, $\mathbb{R}^{N}$, and its complex extension, $\mathrm{C}^{N}$, are examples of vector spaces.

As is well known, any element of a vector space can be expanded in terms of a basis $\left\{e_{i}\right\}$, a set of $N=\operatorname{dim} V$ elements. In addition, the vector space can be equipped with an inner product $(,$,$) that defines the notion of norm and orthogonal-$ ity. By using such an inner product, an orthonormal basis $\left\{e_{i}\right\}$ can be defined by

$$
\left(e_{i}, e_{j}\right)=\delta_{i j} \text {. }
$$

The canonical (column vector) representation for the orthonormal basis is

$$
\left(e_{i}\right)_{j}=\delta_{i j},
$$

where the index $j$ outside the parenthesis is the vector index. In matrix notation the orthogonality relation (3) can be written as

$$
e_{i}^{\top} e_{j}=\delta_{i j},
$$

where $\mathrm{T}$ denotes the transpose. In canonical form, the completeness relation

$$
\sum_{i}^{N} e_{i} e_{i}^{\top}=\mathbf{1}
$$

is obvious. Also, Eq. (6) is invariant under an orthogonal $O(N)$ [unitary $U(N)$ ] transformation of a basis for $V$ $=\mathrm{R}^{N}\left(\mathrm{C}^{N}\right)$.

Once the properties of vector spaces are given, it is easy to show that the set of all $N \times N$ square matrices over the reals, $M_{N}(\mathrm{R})$, or over the complex numbers, $M_{N}(\mathrm{C})$, form a $N^{2}$ dimensional vector space. ${ }^{10}$ In these vector spaces a canonical basis $\left\{e^{i j}\right\}$ is given by the matrices

$$
\left(e^{i j}\right)_{k l}=\delta_{k}^{i} \delta_{l}^{j} \quad(i, j, k, l=1, \ldots, N),
$$

and hence, any $N \times N$ matrix can be expanded as

$$
M=M_{i j} e^{i j},
$$

where the expansion coefficients $M_{i j}=(M)_{i j}$ are the elements of the matrix $M$.

In $M_{N}(\mathbb{R})$ we can define the (positive definite) inner product

$$
(A, B) \equiv \operatorname{Tr}\left[A B^{\top}\right],
$$

for which the canonical basis satisfies

$$
\operatorname{Tr}\left[e^{i j} e^{k l \top}\right]=\delta^{i k} \delta^{j l} .
$$

For $M_{N}(\mathrm{C})$ the transpose operation ( $)^{\top}$ has to be replaced by the hermitian conjugation operation ()$^{\dagger}$.

An equivalent approach is to define a bilinear function on $M_{N}(\mathbb{R})$ without the positive definiteness requirement of an inner product. Thus, instead of defining Eq. (9), we can discard the transpose operation in the definition and regard simply the trace of the product as the relevant bilinear function.
The missing transpose operation can be transferred to the definition of a dual basis $\left\{e_{i j}\right\}: e_{i j} \equiv e^{j i}=e^{i j \top}$. Then the dual basis is the orthogonal counterpart of the basis $\left\{e^{i j}\right\}$ through the trace bilinear. An equivalent way is to regard the trace bilinear between two elements of $\left\{e^{i j}\right\}$ as defining a metric that can be used to lower and raise indices and to interchange the basis with its dual; an analogous construction is found in special relativity when contravariant and covariant fourvectors are defined. An inner product defines, with an appropriate basis, a metric that is the identity matrix. In general there can be nondiagonal or nonpositive definite metrics. This approach will be used to derive the Fierz identities in Sec. III and chiral Fierz identities in Sec. IV.

We use this dual basis to express the expansion coefficient in Eq. (8) as

$$
M_{i j}=\operatorname{Tr}\left[M e_{i j}\right] .
$$

If we substitute Eq. (11) into Eq. (8) and take the respective elements of the matrix, we obtain the trivial relation

$$
\delta_{k m} \delta_{n l}=\left(e_{i j}\right)_{k l}\left(e^{i j}\right)_{n m},
$$

where the summation convention of repeated indices is used here and in the following. This relation follows directly from Eq. (4) and is a completeness relation analogous to Eq. (6). Equation (12) represents an identity in the space of general linear transformations over $M_{N}(\mathbb{R})$. This entire discussion is also valid for $M_{N}(\mathrm{C})$ if one extends the ring from $\mathrm{R}$ to $\mathrm{C}$. Because any linear transformation over $M_{N}(\mathbb{R})$ can be given as a linear combination of transformations of the form

$$
M \rightarrow(A \otimes B) M \equiv A M B^{\top}=(A)_{i j}(B)_{l k}(M)_{j k} e^{i l},
$$

Eq. (12) implies that

$$
\left(e_{i j} \otimes e^{i j}\right) M=\left(e^{i j} \otimes e_{i j}\right) M=M .
$$

Although trivial with this choice of basis, a completeness relation like Eq. (12) is all that is needed to deduce Fierztype identities.

To obtain nontrivial relations, let us take the example of $\mathrm{SU}(2)$ and $\mathrm{SU}(3)$ algebras in the fundamental representation. The commonly used representations for these algebras are the Pauli matrices $\left\{\sigma_{i}\right\}$ and the Gell-Mann matrices $\left\{\lambda_{a}\right\} .11,12$ They form vector spaces and satisfy the orthogonality relations

$$
\begin{aligned}
& \operatorname{Tr}\left[\sigma_{i} \sigma_{j}\right]=2 \delta_{i j} \quad(i, j=1,2,3), \\
& \operatorname{Tr}\left[\lambda_{a} \lambda_{b}\right]=2 \delta_{a b} \quad(a, b=1, \ldots, 8) .
\end{aligned}
$$

Because they are already orthogonal and are Hermitian matrices, there is no need to define a dual basis. However, to span $M_{2}(\mathrm{C})$ and $M_{3}(\mathrm{C})$ the respective identity matrices are needed, because $N^{2}$ basis vectors are required and the Pauli and Gell-Mann matrices are traceless. Then the set $\left\{\mathbf{1}, \sigma_{i}\right\}$ spans $M_{2}(\mathrm{C})$, which means any $2 \times 2$ complex matrix can be expanded in terms of

$$
X=X_{0} \mathbf{1}+X_{i} \sigma^{i}, \quad \sigma^{i}=\sigma_{i},
$$

where

$$
X_{0}=\frac{1}{2} \operatorname{Tr}[X], \quad X_{i}=\frac{1}{2} \operatorname{Tr}\left[X \sigma_{i}\right] .
$$

We substitute Eq. (18) into Eq. (17) and take the general elements 


$$
(X)_{i j}=\frac{1}{2}(X)_{k k} \delta_{i j}+\frac{1}{2}(X)_{l k}\left(\sigma_{m}\right)_{k l}\left(\sigma_{m}\right)_{i j},
$$

and obtain from the coefficients of $(X)_{l k}$, after properly inserting Kronecker deltas, the completeness relation

$$
\delta_{i l} \delta_{k j}=\frac{1}{2} \delta_{i j} \delta_{k l}+\frac{1}{2}\left(\sigma_{m}\right)_{i j}\left(\sigma_{m}\right)_{k l} .
$$

Equation (20) is the identity used to deduce the Fierz identities to Weyl spinors: $\left(\sigma_{\mu}\right)_{i j}\left(\widetilde{\sigma}^{\mu}\right)_{k l}=2 \delta_{i l} \delta_{k j}$, where $\sigma^{\mu}$ $=(\mathbf{1}, \boldsymbol{\sigma})$, and $\widetilde{\sigma}^{\mu}=(\mathbf{1},-\boldsymbol{\sigma})$; the lowering and raising of $\mu$ indices follows the Minkowski metric.

For the Gell-Mann matrices we have similarly,

$$
\frac{1}{2}\left(\lambda_{a}\right)_{i j}\left(\lambda_{a}\right)_{k l}+\frac{1}{3} \delta_{i j} \delta_{k l}=\delta_{i l} \delta_{k j} .
$$

For any fundamental representation of $\mathrm{SU}(\mathrm{N})$ algebra $\left\{T_{a}\right\}$ satisfying $\operatorname{Tr}\left[T_{a} T_{b}\right]=C \delta_{a b}$, we have the completeness relation

$$
\frac{1}{C}\left(T_{a}\right)_{i j}\left(T_{a}\right)_{k l}+\frac{1}{N} \delta_{i j} \delta_{k l}=\delta_{i l} \delta_{k j} .
$$

For the $O(N)$ groups there is no simple relation similar to Eq. (22) because the algebra is formed by $N \times N$ antisymmetric matrices, and thus all symmetric matrices are needed to $\operatorname{span} M_{N}(\mathrm{R})$.

Before introducing Dirac matrices to deduce the Fierz identities, it is better to introduce a clearer notation due to Takahashi, ${ }^{4}$ where we replace the matrix indices by parentheses ( ) and brackets [ ], such that each parenthesis/bracket represents a different index in an unambiguous way. For example, using this notation the relation (22) reads

$$
\frac{1}{C}\left(T_{a}\right)\left[T_{a}\right]+\frac{1}{N}()[]=(][),
$$

where the blank entry means the identity matrix. This notation clearly shows the reordering property.

\section{FIERZ IDENTITIES}

The starting point to derive the usual Fierz identities is the orthogonality relation among the 16 Dirac bilinears ${ }^{13}$ that span $M_{4}(\mathrm{C})$ over $\mathrm{C}$. The 16 Dirac bilinears are usually classified into distinct classes according to their properties under Lorentz transformations ${ }^{3}$ as

$$
\left\{\Gamma^{A}\right\}=\left\{\mathbf{1}, \gamma_{5}, \gamma^{\mu}, \gamma_{5} \gamma^{\mu}, \sigma^{\mu \nu}\right\} \quad(\mu, \nu=0,1,2,3),
$$

where $\mu<\nu$ in $\sigma^{\mu \nu}$ to avoid redundancy. Here the convention used by Itzykson and Zuber ${ }^{15}$ is employed for the gamma matrices:

$$
\begin{aligned}
& \left\{\gamma^{\mu}, \gamma^{\nu}\right\}=2 g^{\mu \nu}, \\
& \sigma^{\mu \nu}=\frac{i}{2}\left[\gamma^{\mu}, \gamma^{\nu}\right], \\
& \gamma^{5}=\gamma_{5}=i \gamma^{0} \gamma^{1} \gamma^{2} \gamma^{3}, \\
& \gamma^{5} \sigma^{\mu \nu}=\frac{i}{2} \varepsilon^{\mu \nu \alpha \beta} \sigma_{\alpha \beta},
\end{aligned}
$$

where $\varepsilon^{0123}=1=-\varepsilon_{0123}$ and $g^{\mu \nu}=\operatorname{diag}(1,-1,-1,-1)$ is the Minkowski metric.

Then, defining a basis $\left\{\Gamma_{A}\right\}$ dual to Eq. (24) as the respective gamma matrices with space-time indices lowered by Minkowski metric, ${ }^{14}$ the orthogonality relation holds:

$$
\operatorname{Tr}\left[\Gamma_{A} \Gamma^{B}\right]=4 \delta_{A}^{B} .
$$

This relation allow us to expand any complex $4 \times 4$ matrix $X$ in terms of the basis (24) as

$$
X=X_{A} \Gamma^{A}, \quad X_{A}=\frac{1}{4} \operatorname{Tr}\left[X \Gamma_{A}\right] .
$$

We combine Eqs. (29) and (30), extract each element of the matrix, and find a completeness relation analogous to Eq. (23):

$$
\text { ( ) }[]=\frac{1}{4}\left(\Gamma_{A}\right]\left[\Gamma^{A}\right)=\frac{1}{4}\left(\Gamma^{A}\right]\left[\Gamma_{A}\right) .
$$

This identity is sufficient to reproduce all possible Fierz identities by appropriately multiplying identity matrices by general matrices $X, Y$ as

$$
\begin{aligned}
(X)[Y] & =(X \mathbf{1})[\mathbf{1} Y]=\frac{1}{4}\left(X \Gamma_{C} Y\right]\left[\Gamma^{C}\right) \\
& =\frac{1}{4^{2}} \operatorname{Tr}\left[X \Gamma_{C} Y \Gamma_{D}\right]\left(\Gamma^{D}\right]\left[\Gamma^{C}\right) .
\end{aligned}
$$

In particular, if $X=\Gamma^{A}$ and $Y=\Gamma^{B}$, Eq. (32) leads to the Fierz identities

$$
\left(\Gamma^{A}\right)\left[\Gamma^{B}\right]=\frac{1}{4^{2}} \operatorname{Tr}\left[\Gamma^{A} \Gamma_{C} \Gamma^{B} \Gamma_{D}\right]\left(\Gamma^{D}\right]\left[\Gamma^{C}\right) .
$$

The only remaining task is to calculate the expansion coefficients which are straightforward gamma matrix traces. ${ }^{15}$ The usual textbook Fierz identities (see, for example, Ref. 11, p. 160) can be found when $\Gamma^{A}$ and $\Gamma^{B}$ in Eq. (33) are chosen to form scalar quantities (under the full Lorentz transformations, including parity) such as $\left(\gamma_{\mu}\right)\left[\gamma^{\mu}\right]$ or Eq. (31) itself. An additional minus sign arises in the Fierz identities (33) when we insert anticommuting fermion fields instead of numerical spinors.

\section{CHIRAL FIERZ IDENTITIES}

The Fierz identities derived in Sec. III are not quite appropriate when treating chirally projected combinations such as

$$
\left(R \gamma^{\mu}\right)\left[L \gamma_{\mu}\right],
$$

where the two chiral projectors are $R=\frac{1}{2}\left(\mathbf{1}+\gamma_{5}\right)$ and $L=\frac{1}{2}(\mathbf{1}$ $-\gamma_{5}$ ) because the expansion (32) applied to Eq. (34) still have some non-null coefficients to be calculated. For nonscalar combinations such as $\left(R \sigma^{\mu \nu}\right)\left[R \gamma_{\nu}\right]$, the number of coefficients to be calculated may become large. Moreover, the relatively simple form of certain chiral Fierz identities such as the form invariants ${ }^{16}$

$$
\begin{aligned}
& \left(R \gamma^{\mu}\right)\left[R \gamma_{\mu}\right]=-\left(R \gamma^{\mu}\right]\left[R \gamma_{\mu}\right), \\
& \left(L \gamma^{\mu}\right)\left[L \gamma_{\mu}\right]=-\left(L \gamma^{\mu}\right]\left[L \gamma_{\mu}\right),
\end{aligned}
$$

suggests a simpler procedure should exist.

A better way to perform Fierz transformations for combinations such as Eq. (34) consists of rederiving Fierz identities using a chiral basis

$$
\left\{\Gamma^{A}\right\}=\left\{R, L, R \gamma^{\mu}, L \gamma^{\mu}, \sigma^{\mu \nu}\right\} \quad(\mu, \nu=0,1,2,3),
$$

where $\mu<\nu$, and its respective dual basis 


$$
\left\{\Gamma_{A}\right\}=\left\{R, L, L \gamma_{\mu}, R \gamma_{\mu}, \frac{1}{2} \sigma_{\mu \nu}\right\} \quad(\mu, \nu=0,1,2,3),
$$

where $\mu<\nu$. Notice that because of the anticommuting nature of $\gamma^{\mu}$ with $\gamma_{5}$ and projector properties, the dual of $R \gamma^{\mu}$ is $L \gamma_{\mu}$. The orthogonality property between the bases (36) and (37) is

$$
\operatorname{Tr}\left[\Gamma_{A} \Gamma^{B}\right]=2 \delta_{A}^{B},
$$

which implies the completeness relation

$$
\begin{aligned}
(\mathrm{)})[]= & \frac{1}{2}\left(\Gamma_{A}\right]\left[\Gamma^{A}\right) \\
= & \frac{1}{2}\left\{(R][R)+(L][L)+\left(R \gamma^{\mu}\right]\left[L \gamma_{\mu}\right),+\left(L \gamma^{\mu}\right]\left[R \gamma_{\mu}\right)\right. \\
& \left.+\left(\frac{1}{2} \sigma^{\mu \nu}\right]\left[\frac{1}{2} \sigma_{\mu \nu}\right)\right\},
\end{aligned}
$$

where the extra $1 / 2$ in the $\sigma^{\mu \nu}$ expansion is inserted to account for the double counting due to the implicit $\mu, \nu$ summation over all values and $\sigma^{\mu \nu}=-\sigma^{\nu \mu}$. Such a completeness relation directly leads to the chiral Fierz identities

$$
\left(\Gamma^{A}\right)\left[\Gamma^{B}\right]=\frac{1}{4} \operatorname{Tr}\left[\Gamma^{A} \Gamma_{C} \Gamma^{B} \Gamma_{D}\right]\left(\Gamma^{D}\right]\left[\Gamma^{C}\right) .
$$

To illustrate the usefulness of the chiral Fierz identities, we apply them to calculate the Fierz transform of the combination (34),

$$
\begin{aligned}
\left(R \gamma^{\mu}\right)\left[L \gamma_{\mu}\right] & =\frac{1}{4} \operatorname{Tr}\left[R \gamma^{\mu} \Gamma_{C} L \gamma_{\mu} \Gamma_{D}\right]\left(\Gamma^{D}\right]\left[\Gamma^{C}\right) \\
& =\frac{1}{4} \operatorname{Tr}\left[R \gamma^{\mu} L L \gamma_{\mu} R\right](R][L)=2(R][L),
\end{aligned}
$$

where the gamma matrix properties ${ }^{15} \quad \gamma^{\mu} \gamma_{\mu}=4 \times \mathbf{1}$, $\gamma^{\mu} \sigma^{\alpha \beta} \gamma_{\mu}=0$, and the trace cyclic property were used. More difficult examples can be worked out, for instance,

$$
\begin{aligned}
\left(R \sigma_{\mu \nu}\right)\left[R \gamma^{\nu}\right]= & \frac{1}{4} \operatorname{Tr}\left[R \sigma_{\mu \nu} R R \gamma^{\nu} L \gamma_{\rho}\right]\left(R \gamma^{\rho}\right][R) \\
& +\frac{1}{4} \operatorname{Tr}\left[R \sigma_{\mu \nu} \frac{1}{2} \sigma_{\alpha \beta} R \gamma^{\nu} L \gamma_{\rho}\right]\left(R \gamma^{\rho}\right]\left[\frac{1}{2} \sigma^{\alpha \beta}\right) \\
= & \frac{3}{2} i\left(R \gamma_{\mu}\right][R)+\frac{1}{2}\left(R \gamma^{\nu}\right]\left[R \sigma_{\nu \mu}\right) .
\end{aligned}
$$

Some labor can be saved by using the trace relation

$$
\begin{aligned}
& \operatorname{Tr}\left[R \sigma_{\mu \nu} \sigma_{\alpha \beta}\right]=2\left(g_{\mu \alpha} g_{\nu \beta}-g_{\mu \beta} g_{\nu \alpha}+i \varepsilon_{\mu \nu \alpha \beta}\right), \\
& \operatorname{Tr}\left[L \sigma_{\mu \nu} \sigma_{\alpha \beta}\right]=2\left(g_{\mu \alpha} g_{\nu \beta}-g_{\mu \beta} g_{\nu \alpha}-i \varepsilon_{\mu \nu \alpha \beta}\right) .
\end{aligned}
$$

One can check the coefficients for the cases $(\mu \nu)=(\alpha \beta)$ and $(\mu \nu \alpha \beta)=(0123)$.

Moreover, the combination of chiral Fierz identities (40) with other completeness relations such as Eq. (21) can be used to decompose four-quark operators carrying other quantum numbers like $\mathrm{SU}(3)$ color. $^{2}$

The simplicity arises because only a few expansion coefficients are nonzero due to the projector properties of $R / L$ and the commuting or anticommuting character of the bilinears with $\gamma_{5}$. Equivalently, $R / L$ projectors reduce the spinor vector space and the resulting projected spinors have to be the same on the two sides of the chiral Fierz identities.

\section{SUMMARY}

The well-known result that the Fierz identities are a consequence of the completeness of the Dirac bilinears as a basis spanning the complex $4 \times 4$ complex matrices was reviewed. Recognizing that bases other than Dirac bilinears are equally possible permitted us to develop a better procedure to calculate the chiral Fierz identities by choosing chirally left/right projected matrices as a basis. The generality of the procedure was stressed and illustrated using the canonical basis of matrix vector spaces, which led to trivial relations in this case.

The usefulness of Fierz-type relations depends on the particular choice of basis and how nearly complete is the set of matrix objects (representations) of interest. The same unified framework was used to derive completeness relations for the generators of the $\mathrm{SU}(\mathrm{N})$ group in the fundamental representation. Other matrix representations or other algebras can be analyzed as well, although they may not be complete and hence the corresponding Fierz-type identities may not be as useful as those presented here.

\section{ACKNOWLEDGMENT}

This work was supported by Conselho Nacional de Desenvolvimento Científico e Tecnológico (CNPq).

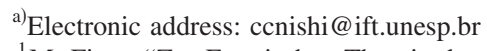

${ }^{1}$ M. Fierz, "Zur Fermischen Theorie des $\beta$-Zerfalls," Z. Phys. 104, $553-$ 565 (1937).

${ }^{2}$ J. F. Donoghue, E. Golowich, and B. R. Holstein, Dynamics of the Standard Model (Cambridge University Press, 1994), pp. 217 and 221.

${ }^{3}$ J. D. Bjorken and S. D. Drell, Relativistic Quantum Mechanics (McGrawHill, New York, 1965).

${ }^{4}$ Y. Takahashi, "The Fierz identities," in Progress in Quantum Field Theory, edited by H. Ezawa and S. Kamefuchi (North-Holland, Amsterdam, 1986), p. 121.

${ }^{5}$ J. F. Nieves and P. B. Pal, "Generalized Fierz identities," Am. J. Phys. 72, 1100-1108 (2004).

${ }^{6}$ R. Mohapatra and P. Pal, Massive Neutrinos in Physics and Astrophysics (World Scientific, Singapore, 1991), pp. 165-70.

${ }^{7}$ The surprising implications and experimental evidences for neutrino oscillations can be found in W. C. Haxton and B. R. Holstein, "Neutrino physics," Am. J. Phys. 68, 15-32 (2000); also W. C. Haxton and B. R. Holstein, "Neutrino physics: An update," Am. J. Phys. 72, 18-24 (2004); see also M. C. Gonzalez-Garcia and Y. Nir, "Neutrino masses and mixing: Evidence and implications," Rev. Mod. Phys. 75, 345-402 (2003).

${ }^{8}$ See any linear algebra book, for example, I. M. Gel'fand, Lectures on Linear Algebra (Interscience, New York, 1961).

${ }^{9}$ Any operation applied to an element in the vector space must result in another element of the vector space.

${ }^{10} M_{N}(\mathrm{C})$ may be considered as a $2 N^{2}$ dimensional vector space if spanned by $N^{2}$ real matrices and $N^{2}$ purely complex matrices with real expansion coefficients only, that is, over the reals R.

${ }^{11}$ See C. Itzykson and J. B. Zuber, Quantum Field Theory (McGraw-Hill, New York, 1980), p. 516, for an explicit representation of Gell-Mann matrices.

${ }^{12} \mathrm{The} \mathrm{SU}(2)$ and $\mathrm{SU}(3)$ generators are $\left\{\frac{1}{2} \sigma_{i}\right\}$ and $\left\{\frac{1}{2} \lambda_{a}\right\}$, respectively.

${ }^{13}$ I will denote as Dirac bilinears the proper bilinears containing the two spinors as well as the associated matrices alone, because the Fierz identities do not depend on the spinors involved.

${ }^{14}$ The lowering of space-time indices is equivalent to the hermitian conjugation operation.

${ }^{15}$ See Ref. 11, Appendix.

${ }^{16}$ These identities were used to get from Eq. (1) to Eq. (2) with a sign difference due to the anticommutation of fermion fields. 